\title{
Interaction of FKBP5 with Childhood Adversity on Risk for Post-Traumatic Stress Disorder
}

\author{
Pingxing Xie ${ }^{1,2}$, Henry R Kranzler ${ }^{3,4}$, James Poling ${ }^{2,5}$, Murray B Stein ${ }^{6,7}$, Raymond F Anton ${ }^{8}$, \\ Lindsay A Farrer, 10,11,12,13 and Joel Gelernter ${ }^{1,2,5, *}$
}

\begin{abstract}
'Department of Genetics, Yale University School of Medicine, New Haven, CT, USA; ${ }^{2}$ VA CT Healthcare Center, West Haven, CT, USA; ${ }^{3}$ Department of Psychiatry, University of Connecticut School of Medicine, Farmington, CT, USA; ${ }^{4}$ Departments of Genetics and Developmental Biology, University of Connecticut School of Medicine, Farmington, CT, USA; ${ }^{5}$ Department of Psychiatry, Yale University School of Medicine, New Haven, CT, USA; 'Department of Psychiatry, University of California, and VA San Diego Healthcare System, San Diego, CA, USA; ${ }^{7}$ Departments of Family and Preventive Medicine, University of California, and VA San Diego Healthcare System, San Diego, CA, USA; ${ }^{8}$ Departments of Psychiatry and Behavioral Sciences, Medical University of South Carolina, Charleston, SC, USA; ' Department of Medicine, Boston University School of Medicine and Public Health, Boston, MA, USA; ${ }^{10}$ Department of Neurology, Boston University School of Medicine and Public Health, Boston, MA, USA; "'Departments of Genetics and Genomics, Boston University School of Medicine and Public Health, Boston, MA, USA; ${ }^{2}$ Department of Epidemiology, Boston University School of Medicine and Public Health, Boston, MA, USA; ${ }^{13}$ Department of Biostatistics, Boston University School of Medicine and Public Health, Boston, MA, USA
\end{abstract}

FKBP5 regulates the cortisol-binding affinity and nuclear translocation of the glucocorticoid receptor. Polymorphisms at the FKBP5 locus have been associated with increased recurrence risk of depressive episodes and rapid response to antidepressant treatment. $A$ recent study showed that FKBP5 genotypes moderated the risk of post-traumatic stress disorder (PTSD) symptoms associated with childhood maltreatment. One thousand one hundred forty-three European Americans (EAs) and 1284 African Americans (AAs) recruited for studies of the genetics of substance dependence were also screened for lifetime PTSD. Four single-nucleotide polymorphisms (SNPs) in FKBP5, rs3800373, rs9296158, rs 1360780, and rs9470080, were genotyped on the complete sample. Logistic regression analyses were performed to explore the interactive effect of FKBP5 polymorphisms and childhood adversity on the risk for PTSD. After correction for multiple testing, childhood adversity significantly increased the risk for PTSD. FKBP5 genotypes were not associated with the development of the disorder. In AAs, one of the SNPs, rs9470080, moderated the risk of PTSD that was associated with childhood abuse. Without childhood adverse experiences, participants with the $T$ genotype of this SNP had the lowest risk for PTSD, whereas they had the highest risk for PTSD after childhood adversity exposure. In addition, in EAs, alcohol dependence was observed to interact with childhood adverse experiences, and also FKBP5 polymorphisms, to increase the risk for PTSD. This study provides further evidence of a gene $\times$ environment effect of FKBP5 and childhood abuse on the risk for PTSD in AAs. Further study is required in other populations.

Neuropsychopharmacology (2010) 35, 1684-1692; doi:10.1038/npp.2010.37; published online I4 April 2010

Keywords: FKBP5; HPA axis; glucocorticoid receptor; childhood adversity; gene-environment interaction; post-traumatic stress disorder

\section{INTRODUCTION}

Post-traumatic stress disorder (PTSD) is a common and frequently disabling psychiatric disorder. Results from the National Comorbidity Survey Replication indicated that the lifetime prevalence of PTSD was 6.8\% (Kessler et al, 2005). PTSD is distinct from other psychiatric disorders in that it requires exposure to a traumatic event. Epidemiological

*Correspondence: Dr J Gelernter, Department of Psychiatry, Yale University School of Medicine, VA CT Healthcare Center, 950 Campbell Avenue, II6A2, West Haven, CT, 06516, USA, Tel: + 203932 57II, Fax: + 203937 474I, E-mail: joel.gelernter@yale.edu Received 30 November 2009; revised 23 February 20 I0; accepted 25 February 2010 studies have provided strong evidence that individuals with adverse experiences during childhood are more sensitive to stressors in their adulthood than those without childhood adversities (Hammen et al, 2000; Kendler et al, 2004). Early adverse experiences greatly increase risk for PTSD (Molnar et al, 2001; Widom, 1999) and other mood and anxiety disorders, such as depression (Chapman et al, 2004; Kessler and Magee, 1993; McCauley et al, 1997; Molnar et al, 2001), panic disorder (Stein et al, 1996) and social phobia (Molnar et al, 2001). Many types of childhood adversity can increase risk for PTSD, including sexual abuse (Molnar et al, 2001; Widom, 1999), physical abuse (Duncan et al, 1996; Widom, 1999), and other traumatic events (Ahmad et al, 2000; Pynoos et al, 1993). 
Hypothalamic-pituitary-adrenal (HPA) axis dysfunction has been implicated in the development of PTSD. The HPA axis is the major neuroendocrine system that regulates stress response in mammals. Following stress exposure, the hypothalamus secretes corticotrophin-releasing hormone and other regulatory neuropeptides, which promote the release of adrenocorticotropic hormone (ACTH) from the pituitary. ACTH stimulates the release of cortisol from the adrenal glands. Cortisol and other glucocorticoids then initiate neural, immune and other biological responses to stress. These hormones also act via a negative feedback loop to the HPA axis to regulate subsequent hormone release (Munck and Guyre, 1986). PTSD patients have been shown in some studies to have lower basal activity of the HPA axis (Bremner et al, 2003; Yehuda et al, 1995), although other studies have not shown this effect (Baker et al, 1999; Maes et al, 1998). Dexamethasone suppression testing has shown that PTSD patients may have higher negative feedback regulation of cortisol than normal controls (de Kloet et al, 2006; Goenjian et al, 1996). In addition, the number and sensitivity of glucocorticoid receptors appear to be increased in mononuclear cells of PTSD patients (Yehuda et al, 2004).

Several studies have shown that childhood abuse is associated with subsequent altered dynamics of the HPA axis in adulthood (Carpenter et al, 2007; Heim et al, 2008). The HPA axis is not mature at birth; adverse early life experiences may influence its development, shaping the basal rhythms and reactivity of the HPA system in adulthood (Gunnar and Donzella, 2002). Considering the importance of the HPA axis in the etiology of mood and anxiety disorders, the effect of childhood adversities on PTSD risk could be mediated by changes in HPA axis function, which could cause maltreated children to be more sensitive to stressors than non-abused individuals (Tarullo and Gunnar, 2006). Indeed, studies have shown that in rodents, maternal behavior changed HPA responses to stress by regulating gene expression through epigenetic programming (Liu et al, 1997; Weaver et al, 2004). A recent postmortem study of suicide victims also showed that childhood abuse decreased glucocorticoid receptor expression by increasing the methylation level of the promoter region (McGowan et al, 2009).

In addition to early environmental adversities, genetic factors and potential gene $\times$ environment interactions are also important in determining HPA axis activity and influencing downstream phenotypes. Unaffected young people with a depressed parent had greater baseline cortisol levels than controls (Mannie et al, 2007), and abnormal responses to the combined dexamethasone/corticotrophinreleasing hormone challenge test (Holsboer et al, 1995; Modell et al, 1998). Genetic contributions to morning cortisol levels were shown to reflect early life experiences (Ouellet-Morin et al, 2009). These findings suggest that gene $\times$ environment interaction affects HPA axis functions. That is, genetic factors may moderate the impact of early life adversity on HPA axis activity, influencing the response to stressors later in life.

Genes involved in the regulation of HPA axis function may associate with PTSD risk. In mammalian cells, in the absence of ligand, the glucocorticoid receptors reside in the cytoplasm. FK506-binding protein 5 (FKBP5 or FKBP51) interacts with the glucocorticoid receptor through heatshock protein 90 (hsp90). Upon ligand binding, FKBP5 is exchanged with FKBP4, which can recruit dynein and promote nuclear translocation of the glucocorticoid receptor (Wochnik et al, 2005). In vitro studies showed that overexpression of human FKBP5 reduced cortisol binding affinity and nuclear translocation of the glucocorticoid receptor, and thus influenced the transcriptional activity of the genes regulated by the steroid hormone-signaling pathway (Davies et al, 2002; Wochnik et al, 2005). In addition, FKBP5 and the glucocorticoid receptor form a negative feedback loop with FKBP5 gene expression induced by glucocorticoid (Binder et al, 2004; Vermeer et al, 2003). Three single nucleotide polymorphisms (SNPs) at the FKBP5 locus, rs4713916, rs1360780, and rs3800373, were associated with increased recurrence of depressive episodes and rapid antidepressant response (Binder et al, 2004; Lekman et al, 2008). FKBP5 gene expression was altered in trauma survivors who eventually experienced PTSD (Segman et al, 2005). A recent study also showed that the expression level of FKBP5 was reduced in PTSD patients (Yehuda et al, 2009), consistent with the increased glucocorticoid receptor sensitivity observed in PTSD patients (Yehuda et al, 2004).

FKBP5 genotype may interact with childhood adversity to influence risk for PTSD. Four SNPs in the FKBP5 gene, rs3800373, rs9296158, rs1360780, and rs9470080, interacted with childhood abuse to modify the severity of adult PTSD symptoms (Binder et al, 2008). Two of the 4 SNPs, rs3800373 and rs1360780, were associated with peri-traumatic dissociation, a well-established risk factor for PTSD, in medically injured children (Koenen et al, 2005).

Based on a strong and consistent body of research, we hypothesized that variation in FKBP5 and its interaction with childhood adversity would be associated with risk for PTSD. Based on previous studies (Binder et al, 2008; Koenen et al, 2005), we examined four SNPs in the FKBP5 gene: rs3800373, rs9296158, rs1360780, and rs9470080.

\section{MATERIALS AND METHODS}

\section{Study Recruitment}

Two thousand four hundred twenty-seven subjects were enrolled in this study. Blood samples were collected during linkage and association studies of the genetics of cocaine, opioid, and alcohol dependence. Four hundred ninety-three participants were recruited as members of families, each of which included at least one affected sibling pair for cocaine or opioid dependence (Gelernter et al, 2005; Gelernter et al, 2006). The remaining samples were collected from cocaine, opioid, or alcohol dependence cases and unaffected controls. All of the subjects were recruited and ascertained using similar methods at four sites: Yale University School of Medicine, University of Connecticut Health Center, Medical University of South Carolina, and McLean Hospital of Harvard Medical School. Written informed consent was obtained from all participants. The institutional review board at each of the participating sites approved the study protocol and consent procedures. 


\section{PTSD Diagnosis and Childhood Adversity Assessment}

All subjects were interviewed by trained interviewers using the Semi-Structured Assessment for Drug Dependence and Alcoholism (SSADDA) (Pierucci-Lagha et al, 2007; PierucciLagha et al, 2005). The SSADDA was used to assess DSM-IV diagnostic criteria for a variety of psychiatric disorders including PTSD. In the PTSD section, the participants were asked whether they had ever experienced or witnessed something so horrible that it would be distressing or upsetting to almost anyone. Those reporting traumatic experiences were then interviewed to assess the presence of PTSD symptoms. The data were scored using a computer algorithm to determine the presence of a lifetime diagnosis of DSM-IV PTSD. The interrater and test-retest reliability $[\kappa]$ of the PTSD diagnosis using the SSADDA has been shown to be 0.59 and 0.76 , respectively (Pierucci-Lagha et al, 2007; Pierucci-Lagha et al, 2005).

The SSADDA Environment section assesses childhood adversity, among other features. Participants were asked whether, by age 13, they had witnessed or experienced a violent crime, had been sexually abused, or had been physically abused. Endorsement of any of these adverse childhood experiences was coded as positive for exposure to childhood adversity.

\section{Genotyping}

For most samples, DNA was obtained from immortalized cell lines; for the rest, DNA was extracted from whole blood or saliva. The four SNPs in the FKBP5 gene, rs3800373, rs9296158, rs1360780, and rs9470080, were genotyped by the TaqMan method using the ABI PRISM 7900 Sequence Detection System (ABI, Foster City, CA, USA) at the Laboratory of Psychiatry Genetics, Yale University School of Medicine. Forty-one ancestry-informative markers (AIMs), including 36 highly ancestry-informative short tandem repeat markers and 5 SNPs (rs1540771, rs2814778, rs1805007, rs1426654 and rs12896399) were genotyped to calculate ancestral proportions for all study participants. Detailed genotyping methods for 37 of the 41 AIMs (including an FY SNP) have been described in detail previously (Yang et al, 2005). The remaining four SNPs were genotyped by the same TaqMan technique as that used for FKBP5 SNPs.

\section{Statistical Analysis}

We used STRUCTURE software (Falush et al, 2003; Pritchard and Rosenberg, 1999; Pritchard et al, 2000) set at 500000 burn-in iterations, followed by 500000 repeats, to analyze the AIMs data, generate ancestral proportions and classify the subjects as European American (EA) or African American (AA).

Logistic regression models were used to examine the association between PTSD diagnosis and the potential explanatory variables. In the primary analyses, the model examined the effects of genotype $\times$ childhood adversity on risk of PTSD, with genotypes for rs3800373, rs9296158, rs1360780, and rs9470080, childhood adversity (coded as 0 for none, 1 for exposure), sex, age, and ancestral proportion scores used as covariates. Then, because participants were recruited during studies of the genetics of opioid, cocaine, and alcohol dependence, we conducted secondary analyses; in addition to the explanatory variables in the primary model, main effects of opioid, cocaine, and alcohol dependence, and their interactions with childhood adversity and the 4 FKBP5 SNP genotypes, were explored. To account for the dependence of the data from individuals in the same family, generalized estimating equation (GEE) analyses were applied to fit the logistic regression models (Zeger and Liang, 1986). EAs and AAs were analyzed separately in all logistic regression models. Categorical variables were analyzed using $\chi^{2}$-tests. All analyses were performed using SAS 9.1. Linkage disequilibrium (LD) plots were constructed using the HAPLOVIEW program (Barrett et al, 2005).

\section{RESULTS}

\section{Demographics}

A total of 2427 subjects were included in this study. Their mean age was 38.6 (SD 10.8) years and $54.4 \%$ of the sample was male. Using AIMs, 1143 subjects were classified as EA and 1284 were classified as AA. The mean (SD) age of EAs (60.0\% male) was 37.6 (11.7) years, and AAs (49.5\% male) was 39.5 (9.9) years. In both populations, women had a higher risk of developing PTSD than men (EA: $\chi_{1}^{2}=18.5$, $P<0.0001$; AA: $\left.\chi_{1}^{2}=4.4, P=0.03\right)$. There was no difference in the rate of lifetime PTSD among EA individuals (14.4\%) and AA individuals $(13.9 \%)\left(\chi_{1}^{2}=0.16, P=0.69\right)$. About half of the subjects were included in a previous study focusing on the interactive effect of a serotonin transporter promoter region polymorphism (5-HTTLPR) and stressful life events on PTSD risk (Xie et al, 2009). The previous study included only individuals who reported having experienced stressful life events. This study included subjects recruited since the previous analyses were conducted and those who did not report any stressful life events.

\section{Genotypes}

Each SNP marker was genotyped at least twice to increase accuracy and call rate. Discordant calls were discarded. The final call rates of rs3800373, rs9296158, rs1360780, and rs 9470080 were $98.3,99.5,99.1$, and $98.9 \%$ in EAs and 98.1, $98.9,99.0$, and $98.8 \%$ in AAs. The minor allele frequencies of rs3800373, rs9296158, rs1360780, and rs 9470080 were 0.30 , $0.33,0.31$, and 0.34 in EAs, and $0.46,0.50,0.44$, and 0.50 in AAs. They were similar to the HapMap data (the HapMap allele frequencies for rs9296158, rs1360780, and rs9470080 are, EA: 0.27, 0.24, 0.28; AA: $0.43,0.39,0.44$. There are no HapMap data for rs3800373), and consistent with a previous study of these SNPs (Binder et al, 2008). The four SNPs span $104 \mathrm{~kb}$ of FKBP5 gene. Results from the HAPLOVIEW software showed that they are in LD in both populations (Figure 1).

\section{Effect of Childhood Adversity on Risk of PTSD}

Three types of childhood adversity were considered in this study: namely, witnessing or experiencing a violent crime, 
a
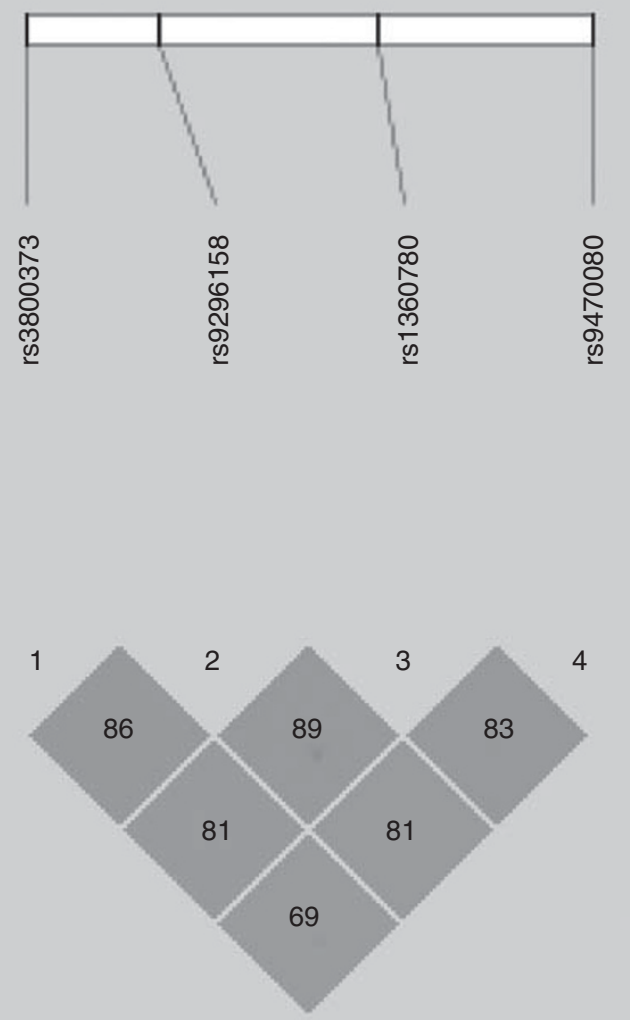

b
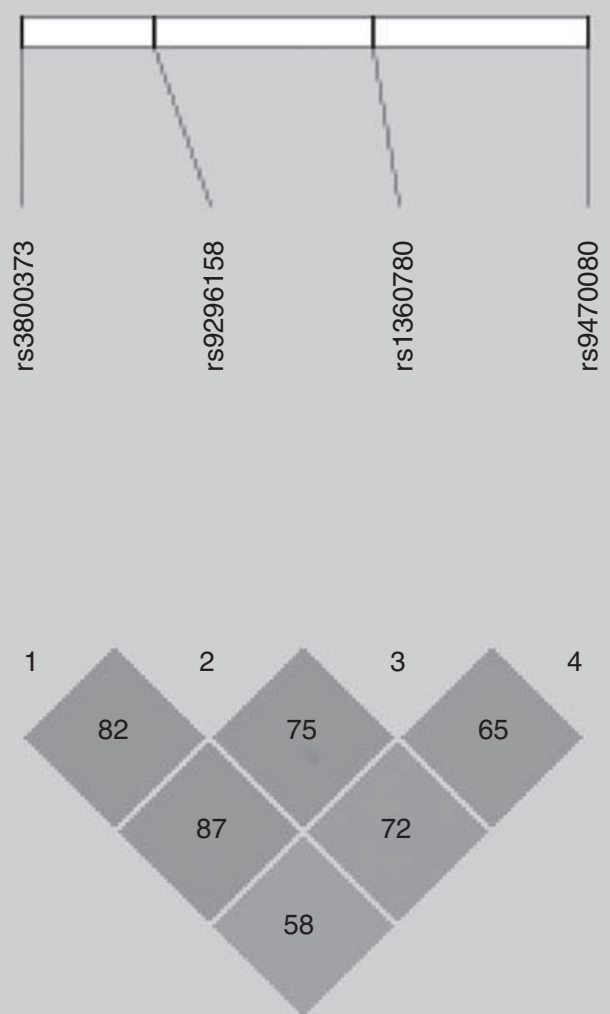

Figure I Linkage disequilibrium plots of rs3800373, rs9296/58, rs I360780, and rs9470080 in EA population (a) and AA population (b). The linkage disequilibrium is depicted as $r^{2}$.

experiencing sexual abuse, and experiencing physical abuse. Among the AAs, $36.1 \%$ reported having experienced at least one type of childhood adversity, which did not differ significantly from that reported by EAs (34.2\%). In the logistic GEE regression model in which we examined the effect of childhood adversity on risk of PTSD, childhood adversity was significantly (positively) associated with risk for PTSD in both EAs and AAs. The average odds ratio (OR) of the effect of childhood adversity on the risk of developing PTSD was 7.69 (95\% confidence interval $[\mathrm{CI}]=4.65-12.72$, $P<0.0001)$ among EAs and $3.30 \quad(95 \% \mathrm{CI}=1.98-5.51$, $P<0.0001)$ among AAs. In addition, the number of types of adversity predicted the development of PTSD. In EAs, $5.9 \%$ of individuals who experienced no childhood adversities developed PTSD, whereas $21.7 \%$ of the subjects with 1 type of childhood adversity were diagnosed with PTSD. In comparison, $45.3 \%$ of those with 2 types of childhood adversities had PTSD, whereas $54.8 \%$ of individuals with all 3 types of childhood adversities developed PTSD. In AAs, the rates of PTSD were 6.6, 18.6, 44.2, and 51.5\% among individuals who experienced $0,1,2$, and 3 types of childhood adversity, respectively.

\section{Effects of FKBP5, and Childhood Adversity $\times$ FKBP5 Genotype on Risk of PTSD}

In the primary analyses, logistic GEE regression showed no significant association between FKBP5 genotypes and PTSD in AAs or EAs (data not shown). When main FKBP5 genotype effects and their interaction effects with childhood adversity were entered into the logistic regression models simultaneously, all four SNPs were significantly associated with the development of PTSD in AAs. After correction for multiple testing, 3 of the 4 SNPs (rs3800373, rs9296158, and rs9470080) remained significant (Bonferroni correction, threshold $P$-value $=0.05 / 4=0.0125$ ). SNP rs 9470080 had the strongest conditional effect. However, in EAs, none of the four SNPs were associated with the development of PTSD when the interactive effect of genotype and childhood adversity was also in the logistic regression model. The results are shown in Table 1.

For the effects of childhood adversity $\times$ FKBP5 genotype on risk for PTSD, results of the primary analyses are shown in Table 1. Before correction for multiple tests, two of the four SNPs in FKBP5 significantly modified the effect of childhood adversity on risk for PTSD in AAs (rs9296158: $\mathrm{OR}=1.69$, $95 \% \mathrm{CI}=1.05-2.71, \quad P=0.03 ; \mathrm{rs} 9470080$ : $\mathrm{OR}=1.96,95 \%$ $\mathrm{CI}=1.24-3.10, P=0.004$ ). (These same two SNPs also were the most significant in the previous study by Binder et al, (2008).) After Bonferroni correction, the signal from rs9470080 remained significant. Without childhood adversity exposure, AA individuals homozygous for the T allele of rs 9470080 had the least chance of developing PTSD compared with subjects with CC and CT genotypes. The differences among the three genotype groups were statistically significant $(P=0.008)$. However, if $\mathrm{T}$ allele homozygotes experienced childhood adversity, they had the highest risk for PTSD (Figure 2), although the difference between TT and the other two genotype groups was not statistically significant. There was no interactive effect of FKBP5 genotypes and childhood adversity in EAs. 
Table I Primary Logistic Regression Analyses Testing G×E Interaction Effects on PTSD Diagnosis with Main Effects (of Both FKBP5 Genotype And Experience of Childhood (Less Than 13 Years of Age) Adversity), and Interactions Entered Simultaneously

\begin{tabular}{|c|c|c|c|c|c|c|}
\hline \multirow{2}{*}{ SNPs } & \multicolumn{2}{|c|}{ Genotype } & \multicolumn{2}{|c|}{ Childhood adversity } & \multicolumn{2}{|c|}{ Genotype $\times$ childhood adversity } \\
\hline & OR (95\% Cl) & $P$-value & OR $(95 \% \mathrm{Cl})$ & $P$-value & OR (95\% Cl) & $P$-value \\
\hline \multicolumn{7}{|c|}{ European American } \\
\hline rs3800373 & $1.15(0.76,1.73)$ & 0.5033 & $8.43(5.08,14.00)$ & $<0.000$ । & $0.74(0.44,1.27)$ & 0.2773 \\
\hline rs9296158 & $0.96(0.64,1.45)$ & 0.8292 & $7.63(4.61,12.65)$ & $<0.000$ । & $0.89(0.52,1.50)$ & 0.6538 \\
\hline \multicolumn{7}{|c|}{ African American } \\
\hline rs3800373 & $0.62(0.43,0.89)$ & 0.0103 & $3.68(2.22,6.10)$ & $<0.000$ । & $1.49(0.93,2.39)$ & 0.0958 \\
\hline rs9296158 & $0.61(0.43,0.88)$ & 0.0081 & $3.15(1.85,5.37)$ & $<0.000$ । & $1.69(1.05,2.7 \mid)$ & 0.0282 \\
\hline rs| 360780 & $0.63(0.44,0.91)$ & 0.0135 & $3.59(2.18,5.92)$ & $<0.000$ । & $1.58(0.98,2.54)$ & 0.0602 \\
\hline
\end{tabular}

RS alleles were typed from the FKBP5 gene.

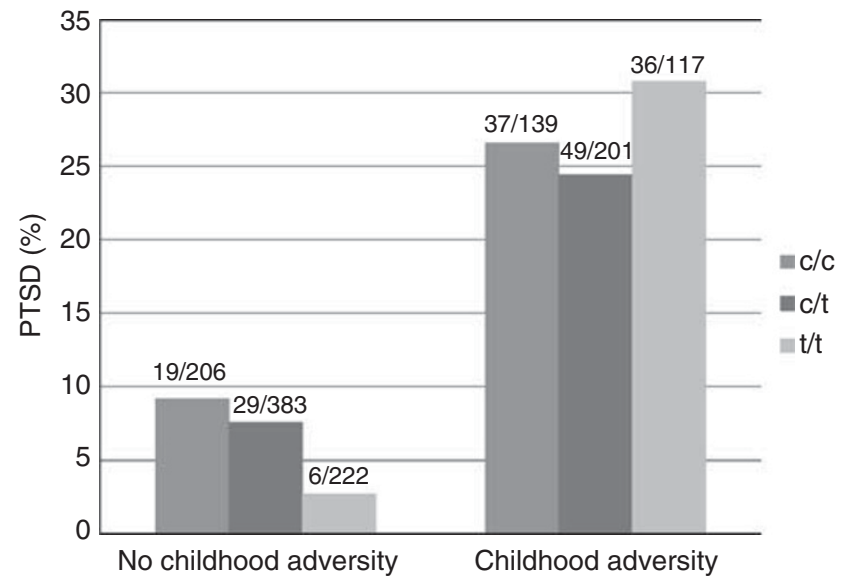

Figure 2 The genotype of rs9470080 interacted with childhood adversity to modify risk for posttraumatic stress disorder (PTSD) in AA individuals. The numbers of subjects with PTSD of the total numbers of participants in the group are shown. Results indicated that AAs homozygous for the T allele at rs9470080 had reduced risk of PTSD without exposure to childhood adversity, and greater risk of PTSD when exposed to childhood adversity, than the other two genotype groups.

To explore the effects of opioid, cocaine, and alcohol dependence on PTSD onset, opioid, cocaine, and alcohol dependence phenotypes, and also their interactions with childhood adversity and the four FKBP5 SNP genotypes were added to the primary logistic GEE regression model. The results are listed in Supplementary Table S1. For AAs, the main effects for cocaine and opioid dependence and all the six interactions were non-significant, so they can be removed from the logistic regression model. The effect for alcohol dependence was significant. When only alcohol dependence was added to the primary model, the results are very similar to the primary results (Table 2). Before correction for multiple tests, rs9296158 and rs9470080 significantly modified the effect of childhood adversity on risk for PTSD ( $\mathrm{rs9296158:} \mathrm{OR}=1.65,95 \% \mathrm{CI}=1.02-2.67$,
$P=0.04 ; \quad$ rs9470080: $\quad \mathrm{OR}=1.91, \quad 95 \% \quad \mathrm{CI}=1.20-3.04$, $P=0.006$ ). After Bonferroni correction, the signal from rs9470080 remained significant. For EAs, results from secondary analyses showed that similar to AAs, alcohol dependence was significantly associated with PTSD onset. In addition, for EAs, the four SNPs in FKBP5 were observed to significantly modify the effect of alcohol and cocaine dependence on risk for PTSD. When the non-significant main effects and interactions were removed from the logistic regression models, no $\mathrm{G} \times \mathrm{E}$ effects of FKBP5 polymorphisms and childhood adversity were observed to modify the risk for PTSD in EAs (Table 2). The results from the secondary analyses are very similar to the primary results.

To exclude the possibility that the gene $\times$ environment interaction effects tested in this study reflect only geneenvironment correlation, the distribution of childhood adversity by genotype groups was examined in both EAs and AAs. By $\chi^{2}$ test, no significant relation was observed in either of the two populations for any of the three childhood adversity types, or for any childhood adversity (Supplementary Table S2).

\section{DISCUSSION}

In this study, we explored the main and interaction effects of FKBP5 genotypes and childhood adversity on the risk for PTSD separately in two populations: EAs and AAs. Four SNPs in the FKBP5 gene, rs3800373, rs9296158, rs1360780, and rs9470080 were chosen based on evidence from previous studies of an interaction effect with environmental factors on PTSD symptoms (Binder et al, 2008; Koenen et al, 2005). Our results (as reported previously in a study that included a subset of the present sample (Xie et al, 2009)) indicated that in both populations, childhood adversity significantly increased the risk for PTSD. FKBP5 genotypes were not associated with the development of this disorder. In AAs, genotypes of rs9296158 and rs 9470080 
Table 2 Results from Secondary Regression Analyses Testing G×E Interaction Effects on PTSD Diagnosis

\begin{tabular}{|c|c|c|c|c|c|c|}
\hline \multirow{2}{*}{ SNPs } & \multicolumn{2}{|c|}{ Genotype } & \multicolumn{2}{|c|}{ Childhood adversity } & \multicolumn{2}{|c|}{ Genotype $\times$ childhood adversity } \\
\hline & OR (95\% Cl) & $P$-value & OR (95\% Cl) & $P$-value & OR (95\% Cl) & $P$-value \\
\hline rs3800373 & I.01 $(0.50,2.05)$ & 0.9722 & I5.8 $(6.38,39.1)$ & $<0.0001$ & $0.70(0.40,1.23)$ & 0.2186 \\
\hline rs9296158 & $0.75(0.37,1.54)$ & 0.4339 & I $4.6(5.93,35.9)$ & $<0.0001$ & $0.83(0.48,1.44)$ & 0.5088 \\
\hline \multicolumn{7}{|c|}{ African American } \\
\hline rs3800373 & $0.63(0.44,0.9 \mid)$ & 0.0146 & $3.21(1.90,5.4 I)$ & $<0.0001$ & $1.49(0.92,2.42)$ & 0.1063 \\
\hline rs9296158 & $0.63(0.44,0.9 \mid)$ & 0.0137 & $2.83(1.64,4.89)$ & 0.0002 & $1.65(1.02,2.67)$ & 0.0405 \\
\hline rs|360780 & $0.64(0.44,0.92)$ & 0.0172 & $3.10(1.86,5.17)$ & $<0.000 \mid$ & $1.61(0.99,2.62)$ & $0.057 \mid$ \\
\hline
\end{tabular}

For European Americans, the independent variables included main effects of FKBP5 genotypes, experience of childhood adversity, alcohol dependence, and cocaine dependence, and interactions of the main effects. For African Americans, the independent variables included main effects of FKBP5 genotypes, experience of childhood adversity, and alcohol dependence, and interactions of genotypes by childhood adversity. Sex, age, and ancestral proportion scores were used as covariates.

moderated the effect of childhood adversity on risk for PTSD. After Bonferroni correction, the rs9470080 remained a significant moderator. Similar to the previous study (Binder et al, 2008), homozygotes for the $\mathrm{T}$ allele at rs9470080 had the lowest risk for PTSD if they never experienced any childhood adversity, but the highest risk to develop PTSD if they were exposed to childhood adversity. However, none of the FKBP5 SNPs modified the effect of childhood adversity in EAs.

These results are consistent with the findings of Binder et al, (2008), which showed an interactive effect of FKBP5 genotype and childhood abuse on risk for PTSD symptoms in a community sample composed mostly of AAs (>95\%). Binder et al, (2008) did not evaluate the interaction effect in EAs. We did not find a significant moderator effect of this gene in EAs. Therefore, this $\mathrm{G} \times \mathrm{E}$ effect may be restricted to AAs. However, among the four SNPs that were previously found to be associated with phenotype, we found that only one had a significant $G \times E$ effect on PTSD risk after correction for multiple comparisons. This SNP, rs9470080, yielded the second smallest $P$-value among the eight SNPs tested in the previous study. The reason for our failure to find the $\mathrm{G} \times \mathrm{E}$ effect for the other three SNPs may be inadequate statistical power, despite the fact that we had access to a reasonably large sample (the study of Binder et al, (2008) included 762 subjects; whereas this study contained 1284 AAs and 1143 EAs). For example, linear regression analysis of PTSD symptom scores might better represent the underlying biological mechanisms than logistic regression models for PTSD diagnosis. To decrease the risk that population stratification would be a confounder in this study, data from 41 AIMs were generated from the complete sample. STRUCTURE software, based on a Bayesian clustering method, was used to divide the participants into two populations. In the logistic regression models, even when the analyses were performed separately for EAs and AAs, ancestry proportion score was used as a covariate, so that population stratification is not a likely confounder in this study.
There are several possibilities to explain the populationspecific effect of FKBP5 genotypes $\times$ childhood adversity on PTSD risk. First, the four SNPs tested in this study may not directly interact with an environmental factor; they may instead reflect the action of variants in LD with these SNPs, which contribute to the $\mathrm{G} \times \mathrm{E}$ effect. FKBP5 LD varies by population (Binder, 2009), providing a possible explanation for failure to detect the signal in one population but not in the other. Second, it is often the case that allele frequencies differ by population, and some polymorphisms are specific to one population or another - in particular, many variants are observed in AAs, but not in EAs (and occasionally, in EAs but not AAs). The specific risk alleles responsible for the observed effect may be rare, or even absent, in EAs. Multiple differences in gene expression and regulation across racial and ethnic groups are other possible factors, which could not be determined directly from a focused investigation as performed here. This is also true of environmental protective factors, such as educational opportunities, access to treatment, and social support. Finally, the association in AAs, but not EAs, could reflect population differences in epistasis.

Results from this study indicated that AAs homozygous for the T allele at rs9470080 had the lowest risk for PTSD if they never experienced any childhood adversity, but the highest risk to develop PTSD if they were exposed to childhood adversity. Based on a previous in vitro study, the TT genotype at rs9470080, which is in strong LD with the TT genotype of rs1360780, was associated with the highest FKBP5 protein expression levels in lymphocytes from healthy individuals (Binder et al, 2004). However, because it is not clear whether these healthy individuals ever experienced childhood adversity, this study provided no information on the expression levels in people with and without childhood adversities as a function of TT genotype at rs9470080. In addition, despite the findings from the in vitro study, to our knowledge, no in vivo studies of the expression levels of different FKBP5 genotypes have been reported. Considering the important role that FKBP5 has in 
the steroid hormone-signaling pathway, in vivo studies and association studies with dense markers are needed to elucidate the nature and contribution of the interaction of FKBP5 with an adverse environment in the etiology of PTSD. In addition, studies examining the relationship between childhood adversity, PTSD diagnosis, and the HPA access should take FKBP5 into account, especially in AA populations.

Because the participants in this study were recruited for studies for the genetics of opioid, cocaine, and alcohol dependence, the effects of these substance dependencies on PTSD onset were explored. In both AA and EA populations, alcohol dependence, but not opioid or cocaine dependence, was significantly associated with PTSD onset. In addition, in EAs, alcohol dependence was observed to interact with childhood adverse experiences, and also FKBP5 polymorphisms, to increase risk for PTSD. Participants carrying the A allele of rs3800373, the G allele of rs9296158, the C allele of rs1360780, and the $C$ allele of rs 9470080 had higher risk for PTSD onset if they were alcohol dependent, compared to participants with other genotypes. This interactive effect was not observed in AAs.

Findings from this study should be interpreted in light of a number of limitations. First, retrospective recall of childhood adverse experiences might be inaccurate or biased. The time interval, significance of events, and personal characteristics could cause recall bias. Second, not all possible types of childhood adversity were covered in this study. For example, familial dysfunction, which is a well-documented risk factor for PTSD (Burton et al, 1994), was not considered in this study. Also, our assessment of childhood adversity was not very detailed; a greater level of detail in relation to that environmental exposure could have led to different conclusions.

In conclusion, this study showed that FKBP5 genotypes, especially rs9470080, interacted with childhood adversity to modify risk for PTSD in AA individuals, a result that is consistent with previous findings.

\section{ACKNOWLEDGEMENTS}

Ann Marie Lacobelle provided technical assistance. We thank Bao-zhu Yang, PhD for helpful comments on the article. We thank the individuals and families participating in this work and the interviewers at all the participating sites who conducted the diagnostic interviews. Kathleen Brady, MD, PhD of the Medical University of South Carolina and Roger Weiss, MD of McLean Hospital and Harvard Medical School oversaw study recruitment at their respective sites. This study was supported by NIH Grants R01 DA12690, R01 DA12849, K24 DA15105, K24 AA013736, K24 DA022288, and R01 AA11330; and the VA National Center for PTSD Research.

\section{CONFLICT OF INTEREST}

Dr Kranzler has received consulting fees from OrthoMcNeil Pharmaceuticals (Raritan, NJ), H. Lundbeck A/S (Copenhagen, Denmark), Forest Pharmaceuticals (St Louis, MO), elbion NV (Leuven, Belgium), Sanofi-Aventis (Bridgewater, NJ), Solvay Pharmaceuticals (Brussels, Belgium), and
Alkermes Inc. (Cambridge, MA). He has received research support from Ortho-McNeil Pharmaceuticals, Bristol-Myers Squibb Company (New York, NY), and Merck \& Co. Inc., (Whitehouse Station, NJ) and honoraria from Forest Pharmaceuticals and Alkermes Inc. Dr Stein declares that he has in the past 3 years received Research Support from: National Institute of Mental Health; Veteran's Affairs Research Program; Department of Defense; Eli Lilly and Company; GlaxoSmithKline; Hoffmann-La Roche. And in the past 3 years has been a Consultant for: AstraZeneca; BrainCells Inc.; Bristol-Myers Squibb; Comprehensive NeuroScience; Eli Lilly and Company; Forest Laboratories; Hoffmann-La Roche Pharmaceuticals; Jazz Pharmaceuticals; Johnson \& Johnson; Mindsite; Pfizer; and Sepracor. Dr Anton reports being a consultant for Sanofi Aventis, Eli Lilly, Merck, Organon, Hythiam, Johnson \& Johnson, and GlaxoSmithKline; serving as a scientific advisory board member for Sanofi Aventis, Merck, Hythiam, Novartis, and Johnson \& Johnson; and receiving grant support from Eli Lily, Merck, Hythiam, Janssen, Schering Plough, Lundbeck, Alkermes, GlaxoSmithKline, and Johnson \& Johnson. Dr Gelernter reports that he has received compensation for professional services in the previous 3 years from the following entities: Yale University School of Medicine, Veterans Affairs Healthcare System (VA) and the National Institutes of Health (NIAAA, NIDA, and NIMH) and related to academic lectures and editorial functions in various scientific venues (including the ACNP). Ms Xie and Drs Poling and Farrer report no conflicts of interest.

\section{REFERENCES}

Ahmad A, Sofi MA, Sundelin-Wahlsten V, von Knorring AL (2000). Posttraumatic stress disorder in children after the military operation 'Anfal' in Iraqi Kurdistan. Eur Child Adolesc Psychiatry 9: 235-243.

Baker DG, West SA, Nicholson WE, Ekhator NN, Kasckow JW, Hill KK et al (1999). Serial CSF corticotropin-releasing hormone levels and adrenocortical activity in combat veterans with posttraumatic stress disorder. Am J Psychiatry 156: 585-588.

Barrett JC, Fry B, Maller J, Daly MJ (2005). Haploview: analysis and visualization of LD and haplotype maps. Bioinformatics (Oxford, England) 21: 263-265.

Binder EB (2009). The role of FKBP5, a co-chaperone of the glucocorticoid receptor in the pathogenesis and therapy of affective and anxiety disorders. Psychoneuroendocrinology 34(Suppl 1): S186-S195.

Binder EB, Bradley RG, Liu W, Epstein MP, Deveau TC, Mercer KB et al (2008). Association of FKBP5 polymorphisms and childhood abuse with risk of posttraumatic stress disorder symptoms in adults. JAMA 299: 1291-1305.

Binder EB, Salyakina D, Lichtner P, Wochnik GM, Ising M, Putz B et al (2004). Polymorphisms in FKBP5 are associated with increased recurrence of depressive episodes and rapid response to antidepressant treatment. Nat Genet 36: 1319-1325.

Bremner JD, Vythilingam M, Anderson G, Vermetten E, McGlashan T, Heninger G et al (2003). Assessment of the hypothalamic-pituitary-adrenal axis over a 24 -h diurnal period and in response to neuroendocrine challenges in women with and without childhood sexual abuse and posttraumatic stress disorder. Biol Psychiatry 54: 710-718.

Burton D, Foy D, Bwanausi C, Johnson J, Moore L (1994). The relationship between traumatic exposure, family dysfunction, and post-traumatic stress symptoms in male juvenile offenders. J Trauma Stress 7: 83-93. 
Carpenter LL, Carvalho JP, Tyrka AR, Wier LM, Mello AF, Mello MF et al (2007). Decreased adrenocorticotropic hormone and cortisol responses to stress in healthy adults reporting significant childhood maltreatment. Biol Psychiatry 62: 1080-1087.

Chapman DP, Whitfield CL, Felitti VJ, Dube SR, Edwards VJ, Anda RF (2004). Adverse childhood experiences and the risk of depressive disorders in adulthood. J Affect Disord 82: 217-225.

Davies TH, Ning YM, Sanchez ER (2002). A new first step in activation of steroid receptors: hormone-induced switching of FKBP51 and FKBP52 immunophilins. J Biol Chem 277: 4597-4600. de Kloet CS, Vermetten E, Geuze E, Kavelaars A, Heijnen CJ, Westenberg HG (2006). Assessment of HPA-axis function in posttraumatic stress disorder: pharmacological and nonpharmacological challenge tests, a review. J Psychiatric Res 40: 550-567.

Duncan RD, Saunders BE, Kilpatrick DG, Hanson RF, Resnick HS (1996). Childhood physical assault as a risk factor for PTSD, depression, and substance abuse: findings from a national survey. Am J Orthopsychiatry 66: 437-448.

Falush D, Stephens M, Pritchard JK (2003). Inference of population structure using multilocus genotype data: linked loci and correlated allele frequencies. Genetics 164: 1567-1587.

Gelernter J, Panhuysen C, Weiss R, Brady K, Hesselbrock V, Rounsaville B et al (2005). Genomewide linkage scan for cocaine dependence and related traits: significant linkages for a cocainerelated trait and cocaine-induced paranoia. Am J Med Genet B Neuropsychiatr Genet 136B: 45-52.

Gelernter J, Panhuysen C, Wilcox M, Hesselbrock V, Rounsaville B, Poling J et al (2006). Genomewide linkage scan for opioid dependence and related traits. Am J Hum Genet 78: 759-769.

Goenjian AK, Yehuda R, Pynoos RS, Steinberg AM, Tashjian M, Yang RK et al (1996). Basal cortisol, dexamethasone suppression of cortisol, and MHPG in adolescents after the 1988 earthquake in Armenia. Am J Psychiatry 153: 929-934.

Gunnar MR, Donzella B (2002). Social regulation of the cortisol levels in early human development. Psychoneuroendocrinology 27: 199-220.

Hammen C, Henry R, Daley SE (2000). Depression and sensitization to stressors among young women as a function of childhood adversity. J Consult Clin Psychol 68: 782-787.

Heim C, Newport DJ, Mletzko T, Miller AH, Nemeroff CB (2008). The link between childhood trauma and depression: insights from HPA axis studies in humans. Psychoneuroendocrinology 33: 693-710.

Holsboer F, Lauer CJ, Schreiber W, Krieg JC (1995). Altered hypothalamic-pituitary-adrenocortical regulation in healthy subjects at high familial risk for affective disorders. Neuroendocrinology 62: 340-347.

Kendler KS, Kuhn JW, Prescott CA (2004). Childhood sexual abuse, stressful life events and risk for major depression in women. Psychol Med 34: 1475-1482.

Kessler RC, Berglund P, Demler O, Jin R, Merikangas KR, Walters EE (2005). Lifetime prevalence and age-of-onset distributions of DSM-IV disorders in the National Comorbidity Survey Replication. Arch Gen Psychiatry 62: 593-602.

Kessler RC, Magee WJ (1993). Childhood adversities and adult depression: basic patterns of association in a US national survey. Psychol Med 23: 679-690.

Koenen KC, Saxe G, Purcell S, Smoller JW, Bartholomew D, Miller A et al (2005). Polymorphisms in FKBP5 are associated with peritraumatic dissociation in medically injured children. Mol Psychiatry 10: 1058-1059.

Lekman M, Laje G, Charney D, Rush AJ, Wilson AF, Sorant AJ et al (2008). The FKBP5-gene in depression and treatment response-an association study in the sequenced treatment alternatives to relieve depression (STAR*D) cohort. Biol Psychiatry 63: 1103-1110.
Liu D, Diorio J, Tannenbaum B, Caldji C, Francis D, Freedman A et al (1997). Maternal care, hippocampal glucocorticoid receptors, and hypothalamic-pituitary-adrenal responses to stress. Science (New York, NY) 277: 1659-1662.

Maes M, Lin A, Bonaccorso S, van Hunsel F, Van Gastel A, Delmeire L et al (1998). Increased 24-h urinary cortisol excretion in patients with post-traumatic stress disorder and patients with major depression, but not in patients with fibromyalgia. Acta psychiatrica Scandinavica 98: 328-335.

Mannie ZN, Harmer CJ, Cowen PJ (2007). Increased waking salivary cortisol levels in young people at familial risk of depression. Am J Psychiatry 164: 617-621.

McCauley J, Kern DE, Kolodner K, Dill L, Schroeder AF, DeChant HK et al (1997). Clinical characteristics of women with a history of childhood abuse: unhealed wounds. JAMA 277: $1362-1368$

McGowan PO, Sasaki A, D’Alessio AC, Dymov S, Labonte B, Szyf M et al (2009). Epigenetic regulation of the glucocorticoid receptor in human brain associates with childhood abuse. Nat Neurosci 12: $342-348$.

Modell S, Lauer CJ, Schreiber W, Huber J, Krieg JC, Holsboer F (1998). Hormonal response pattern in the combined DEX-CRH test is stable over time in subjects at high familial risk for affective disorders. Neuropsychopharmacology 18: 253-262.

Molnar BE, Buka SL, Kessler RC (2001). Child sexual abuse and subsequent psychopathology: results from the National Comorbidity Survey. Am J Public Health 91: 753-760.

Munck A, Guyre PM (1986). Glucocorticoid physiology, pharmacology and stress. Adv Exp Med Biol 196: 81-96.

Ouellet-Morin I, Dionne G, Perusse D, Lupien SJ, Arseneault L, Barr RG et al (2009). Daytime cortisol secretion in 6-month-old twins: genetic and environmental contributions as a function of early familial adversity. Biol Psychiatry 65: 409-416.

Pierucci-Lagha A, Gelernter J, Chan G, Arias A, Cubells JF, Farrer L et al (2007). Reliability of DSM-IV diagnostic criteria using the semi-structured assessment for drug dependence and alcoholism (SSADDA). Drug and Alcohol Depend 91: 85-90.

Pierucci-Lagha A, Gelernter J, Feinn R, Cubells JF, Pearson D, Pollastri A et al (2005). Diagnostic reliability of the Semistructured Assessment for Drug Dependence and Alcoholism (SSADDA). Drug and Alcohol Depend 80: 303-312.

Pritchard JK, Rosenberg NA (1999). Use of unlinked genetic markers to detect population stratification in association studies. Am J Hum Genet 65: 220-228.

Pritchard JK, Stephens M, Donnelly P (2000). Inference of population structure using multilocus genotype data. Genetics 155: 945-959.

Pynoos RS, Goenjian A, Tashjian M, Karakashian M, Manjikian R, Manoukian G et al (1993). Post-traumatic stress reactions in children after the 1988 Armenian earthquake. $\mathrm{Br} J$ Psychiatry 163: 239-247.

Segman RH, Shefi N, Goltser-Dubner T, Friedman N, Kaminski N, Shalev AY (2005). Peripheral blood mononuclear cell gene expression profiles identify emergent post-traumatic stress disorder among trauma survivors. Mol Psychiatry 10: 500-513, 425

Stein MB, Walker JR, Anderson G, Hazen AL, Ross CA, Eldridge $\mathrm{G}$ et al (1996). Childhood physical and sexual abuse in patients with anxiety disorders and in a community sample. Am J Psychiatry 153: 275-277.

Tarullo AR, Gunnar MR (2006). Child maltreatment and the developing HPA axis. Hormones Behav 50: 632-639.

Vermeer H, Hendriks-Stegeman BI, van der Burg B, van BuulOffers SC, Jansen M (2003). Glucocorticoid-induced increase in lymphocytic FKBP51 messenger ribonucleic acid expression: a potential marker for glucocorticoid sensitivity, potency, and bioavailability. J Clin Endocrinol Metabol 88: 277-284. 
Weaver IC, Cervoni N, Champagne FA, D’Alessio AC, Sharma S, Seckl JR et al (2004). Epigenetic programming by maternal behavior. Nat Neurosci 7: 847-854.

Widom CS (1999). Posttraumatic stress disorder in abused and neglected children grown up. Am J Psychiatry 156: 1223-1229.

Wochnik GM, Ruegg J, Abel GA, Schmidt U, Holsboer F, Rein T (2005). FK506-binding proteins 51 and 52 differentially regulate dynein interaction and nuclear translocation of the glucocorticoid receptor in mammalian cells. J Biol Chem 280: 4609-4616.

Xie P, Kranzler HR, Poling J, Stein MB, Anton RF, Brady K et al (2009). Interactive effect of stressful life events and the serotonin transporter 5-HTTLPR genotype on posttraumatic stress disorder diagnosis in 2 independent populations. Arch Gen Psychiatry 66: 1201-1209.

Yang BZ, Zhao H, Kranzler HR, Gelernter J (2005). Practical population group assignment with selected informative markers: characteristics and properties of Bayesian clustering via STRUCTURE. Genet Epidemiol 28: 302-312.

Yehuda R, Cai G, Golier JA, Sarapas C, Galea S, Ising M et al (2009). Gene expression patterns associated with posttraumatic stress disorder following exposure to the World Trade Center attacks. Biol Psychiatry 66: 708-711.

Yehuda R, Golier JA, Yang RK, Tischler L (2004). Enhanced sensitivity to glucocorticoids in peripheral mononuclear leukocytes in posttraumatic stress disorder. Biol Psychiatry 55: $1110-1116$.

Yehuda R, Kahana B, Binder-Brynes K, Southwick SM, Mason JW, Giller EL (1995). Low urinary cortisol excretion in Holocaust survivors with posttraumatic stress disorder. Am J Psychiatry 152: 982-986.

Zeger SL, Liang KY (1986). Longitudinal data analysis for discrete and continuous outcomes. Biometrics 42: 121-130.

Supplementary Information accompanies the paper on the Neuropsychopharmacology website (http://www.nature.com/npp) 